\title{
Effect of Platelet Transfusion on Clot Strength in Dengue Fever with Thrombocytopenia Related Bleeding: A Thromboelastography-Based Study
}

\author{
Varun Sundar ${ }^{\mathrm{a}}$ Emmanuel Bhaskar ${ }^{\mathrm{b}}$ \\ ${ }^{a}$ Community Research Network, Chennai, India; ${ }^{b}$ Department of Medicine, Sri Ramachandra Medical College and \\ Research Institute, Chennai, India
}

\author{
Keywords \\ Dengue fever · Platelet transfusion · Thromboelastography · \\ Clot strength
}

\begin{abstract}
Platelet transfusions are often administered in dengue illness complicated by thrombocytopenia-related bleeding. However, whether this practice results in an improvement of clot strength is not clear. This study assessed the impact of platelet transfusion on the control of bleeding and improvement in clot strength as assessed by thromboelastography among 74 patients with dengue. The effect of either weightbased random donor platelets or 1 unit of single donor aphaeresis units was studied. Transfusion of weight-based random donor platelets resulted in a mean platelet count increase of 10,210 cells per $\mathrm{mm}^{3}$ at $24 \mathrm{~h}$ from pretransfusion values, which reached marginal statistical significance $(p=$ 0.031). Patients who received single donor platelets achieved a mean platelet increment of 22,874 cells per $\mathrm{mm}^{3}$ at $24 \mathrm{~h}$, and the difference observed had a high statistical significance $(p<0.001)$. However, no significant improvement in clot strength was observed in thromboelastography. The mean increment in maximum amplitude value at $24 \mathrm{~h}$ was only $2 \mathrm{~mm}$ in the random donor platelet group and $5 \mathrm{~mm}$ in the single donor group, both of which did not reach statistical significance. Furthermore, the majority of patients had ongoing bleeding despite the administration of platelets.
\end{abstract}

\section{KARGER}

() 2019 S. Karger AG, Basel
This study observed that platelet transfusion in dengue patients with bleeding complication improved the absolute platelet count with no improvement in clot strength.

(c) 2019 S. Karger AG, Basel

Dengue fever is a mosquito-borne viral illness associated with severe thrombocytopenia leading to increased utilization of platelet transfusions [1]. While thrombocytopenia is the major hematological abnormality resulting from the illness, additional factors contribute to bleeding in dengue [2]. Though platelet transfusion is administered to improve platelet counts among dengue patients with bleeding, there is lack of evidence on the impact of such transfusions on bleeding and laboratory assessment of clot strength. Increments in platelet count ranging from 7,000 to 18,000 cells per $\mathrm{mm}^{3}$ occurs as a result of single donor or pooled random donor platelet transfusion [3]. However, whether this practice results in an improvement of platelet function and hence clot strength is not clear. Thromboelastography (TEG) is a laboratory method to assess the viscoelastic property of whole blood during the process of clotting [4]. It is used for hemostatic monitoring in the setting of trauma and major surgery $[5,6]$. The method incorporates assessment of the tensile force which occurs between the clotting blood and a pin as a result of interaction between glycoprotein IIb/IIIa receptors and fibrin [4]. Important

Dr. Varun Sundar

Community Research Network

7 Manickavasagar Street

Gandhi Nagar, Saligramam, Chennai 600093 (India)

E-Mail sundarvrn@yahoo.in 
parameters in TEG are the $\mathrm{R}$ time (period for a $2-\mathrm{mm}$ amplitude in the clot) which depends on clotting factors, $\mathrm{K}$ time (period for 2- to $20-\mathrm{mm}$ amplitude) which depends on clotting factors and fibrinogen, $\alpha$ angle (slope between $\mathrm{R}$ and $\mathrm{K}$ ) which gives the relation between $\mathrm{R}$ and $\mathrm{K}$ time, MA (maximum amplitude of clot strength) which is contributed by platelet function and fibrinogen, and LT (clot lysis time after 30 and $60 \mathrm{~min}$ ) which depends on the function of fibrinolysis [4]. Of these parameters, MA reflects the contribution of platelet function to final clot strength when clotting factors and fibrinogen are normal [4]. This study was initiated to assess the impact of platelet transfusion on the resolution of bleeding and its effect on clot strength as assessed by TEG among dengue patients with isolated thrombocytopenia and bleeding complications.

Participants were aged $\geq 18$ years and were diagnosed with dengue as per the WHO 2009 definition, were seropositive, and had bleeding complications requiring transfusion of either random donor platelets (1 unit for every $10 \mathrm{~kg}$ of patient body weight) or 1 unit of single donor platelets [7]. We excluded participants: (a) with a prior hematological disorder, (b) with splenomegaly due to any cause, (c) with chronic liver disease, (d) on anti-platelet or anticoagulant medications, (e) on hemodialysis, (f) with high $\mathrm{R}$ and $\mathrm{K}$ values in the baseline TEG, (g) with serum fibrinogen $<200 \mathrm{mg} / \mathrm{dL}$, (h) with baseline hemoglobin less than $11 \mathrm{~g} / \mathrm{dL}$ for women and $12 \mathrm{~g} / \mathrm{dL}$ for men, and (i) with lack of bleeding at presentation. Platelet products were received from a blood bank accredited with national quality boards.

Kaolin-activated TEG as per instrument recommendation was performed using a TEG 5000 Hemostasis analyzer system (Haemonetics Corporation, Braintree, MA, USA) for all participants just before the initiation of platelet transfusion and repeated again at 1, 4, 12, and $24 \mathrm{~h}$ after the completion of platelet transfusion. Maximum amplitude (MA), a TEG indicator of clot strength (when fibrinogen and early TEG indicators like $\mathrm{R}$ and $\mathrm{K}$ are normal) was the primary TEG parameter compared between pre- and posttransfusion TEG. Platelet counts prior to transfusion and at intervals of $1,4,12$, and $24 \mathrm{~h}$ were assessed by an automated method which was confirmed with manual counting. Written informed consent was obtained from patients for two additional blood samples (4- and 12-h platelet counts and TEG) apart from the usual monitoring samples. Ethics committee approval was not required since the observation was a case series study design where evaluation and treatment followed the usual standard of care.

Categorical variables are expressed as number (\%) and continuous variables are expressed as the mean \pm SD. Differences between pretransfusion and posttransfusion platelet count and MA values were analyzed with
Table 1. Baseline characteristics and coagulation parameters of study participants $(n=74)$

\begin{tabular}{lc}
\hline Variable & $\begin{array}{c}\text { Number }(\%) \\
\text { or mean } \pm \text { SD }\end{array}$ \\
\hline Gender & \\
Male & $41(55)$ \\
Female & $31(45)$ \\
Age in years & \\
Male & $28 \pm 9.4$ \\
Female & $26 \pm 6.6$ \\
Day of illness & $6 \pm 1.5$ \\
Nature of bleeding & \\
Oral mucosal & $20(27)$ \\
Epistaxis & $6(08)$ \\
Hematuria & $20(27)$ \\
Hematemesis & $12(16)$ \\
Melena & $8(11)$ \\
Menorrhagia & $8(11)$ \\
Coagulation profile & \\
INR & $0.86 \pm 0.12$ \\
aPTT, s & $28 \pm 3.25$ \\
Fibrinogen level, mg/dL & $296 \pm 42.5$ \\
\hline
\end{tabular}

INR, international normalized ratio; aPTT, activated partial thromboplastin time.

one-way analysis of variance (ANOVA) followed post hoc comparison between groups using the Tukey HSD test. A $p$ value $<0.05$ was considered statistically significant. The analysis was conducted with SPSS version 19.1 .

Fifty-two (70\%) patients received random donor platelets and $22(30 \%)$ received single donor platelets. Table 1 describes the baseline characteristics and coagulation profile of the study participants. All 6 patients with epistaxis received nasal packing which controlled the bleeding before the initiation of platelet transfusion. Six out of 20 participants who had oral mucosal bleeding improved with oral rinse with diluted adrenaline, while the remaining 14 had persistent bleeding for a mean duration of $18.5 \mathrm{~h}$ after the infusion of platelets. Ten out of 12 participants who had hematemesis had persistent hemorrhagic nasogastric tube aspirate until endoscopic adrenaline lavage was given. Melena persisted in 6 out of 8 patients for a mean period of $36.8 \mathrm{~h}$ after platelet transfusion. Hematuria persisted for the 20 patients for a mean period of $22.2 \mathrm{~h}$. All the 8 patients who had menorrhagia had persistent bleeding for a mean period of $26.4 \mathrm{~h}$ and improved a day after oral hormonal therapy. Immediate control of bleeding after platelet transfusion occurred only in 2 patients who had hematemesis (as evidenced by clear nasogastric tube aspirate).

Table 2 describes the baseline platelet and TEG parameters, increments in platelet count, and MA values 
Table 2. Platelet count and TEG parameters before and after transfusion

\begin{tabular}{|c|c|c|c|c|}
\hline $\begin{array}{l}\text { Platelet count and TEG } \\
\text { parameters }\end{array}$ & $\begin{array}{l}\text { Random donor } \\
\text { platelets } \\
(n=52)\end{array}$ & $p$ value $^{1}$ & $\begin{array}{l}\text { Single donor } \\
\text { platelets } \\
(n=22)\end{array}$ & $p$ value $^{1}$ \\
\hline \multicolumn{5}{|l|}{ Platelet Count, cells $/ \mathrm{mm}^{3}$} \\
\hline Pretransfusion & $22,820 \pm 12,218$ & & $16,760 \pm 7,220$ & \\
\hline Increment at $1 \mathrm{~h}$ & $9,000 \pm 3,560$ & 0.027 & $24,200 \pm 2,660$ & $<0.001$ \\
\hline Increment at $4 \mathrm{~h}$ & $12,360 \pm 4,680$ & 0.018 & $26,210 \pm 3,582$ & $<0.001$ \\
\hline Increment at $12 \mathrm{~h}$ & $11,220 \pm 2,218$ & 0.021 & $25,310 \pm 3,640$ & $<0.001$ \\
\hline Increment at $24 \mathrm{~h}$ & $10,210 \pm 3,560$ & 0.028 & $22,874 \pm 2,684$ & $<0.001$ \\
\hline Baseline $\mathrm{R}$ value in TEG (normal $4-8 \mathrm{~min}$ ), min & $5.4 \pm 0.8$ & & $5.2 \pm 0.6$ & \\
\hline Baseline $\mathrm{K}$ value in TEG (normal $0-4 \mathrm{~min}$ ), min & $2.1 \pm 0.6$ & & $1.9 \pm 0.5$ & \\
\hline Baseline angle in TEG (normal $\left.47-74^{\circ}\right){ }^{\circ}$ & $54 \pm 12$ & & $48 \pm 10$ & \\
\hline \multicolumn{5}{|c|}{ Maximum amplitude value in TEG (normal $54-72 \mathrm{~mm}$ ), $\mathrm{mm}$} \\
\hline Pretransfusion & $26 \pm 8$ & & $22 \pm 4$ & \\
\hline Value at $1 \mathrm{~h}$ & $28 \pm 6$ & 0.47 & $26 \pm 3$ & 0.33 \\
\hline Value at $4 \mathrm{~h}$ & $30 \pm 4$ & 0.42 & $28 \pm 4$ & 0.21 \\
\hline Value at $12 \mathrm{~h}$ & $29 \pm 3$ & 0.39 & $26 \pm 4$ & 0.39 \\
\hline Value at $24 \mathrm{~h}$ & $28 \pm 4$ & 0.44 & $27 \pm 4$ & 0.44 \\
\hline
\end{tabular}

in each group. Participants who received random donor platelets had a mean platelet count increment of 10,210 cells per $\mathrm{mm}^{3}$ at $24 \mathrm{~h}$ from the pretransfusion value, which reached marginal statistical significance $(p=$ 0.031 ). The group which received single donor platelets achieved a mean platelet increment of 22,874 cells per $\mathrm{mm}^{3}$ at $24 \mathrm{~h}$, with the difference observed from the pretransfusion value having a high statistical significance $(p<0.001)$. However, improvement in clot strength as indicated by an improvement in the MA value in TEG was not observed. The mean increment in MA value at $24 \mathrm{~h}$ was only $2 \mathrm{~mm}$ in the random donor platelet group and $5 \mathrm{~mm}$ in the single donor group, both of which did not reach statistical significance. Of the 2 patients (with hematemesis) who appeared to have benefitted from platelet transfusion the MA value increment was only $4 \mathrm{~mm}$ (from baseline MA of $24 \mathrm{~mm}$ ) and $6 \mathrm{~mm}$ (from baseline MA of $28 \mathrm{~mm}$ ), respectively. Follow-up R, K, and angle values remained normal after transfusion for all participants.

The inference from this preliminary study with a small sample of dengue patients who had thrombocytopenia-related bleeding (since we excluded patients with a multifactorial cause for bleeding) is that platelet transfusion, despite producing a $50-100 \%$ increment in platelet count, has no significant impact in clinical bleeding and clot strength as assessed by TEG. The majority of patients continued to have persistent bleeding despite platelet transfusion. The study raises a larger question of the status of platelet function in both random and single apheresis platelets prior to transfusion. It appears that the transfused platelets do not have adequate function to improve clot strength or possible in vivo factors, which do not allow the proper function of transfused platelets. Future studies can incorporate assessment of platelet function in platelet preparations prior to transfusion to determine whether there is a pretransfusion functional defect in platelets. Our study has the significant limitation of not comparing TEG observations with better laboratory studies for platelet function like platelet aggregometry, secretory studies, and flow cytometry [8]. With literature evidence emerging on the delay in platelet recovery in patients receiving platelet transfusions, further studies which analyze platelet function prior to and following transfusion will provide more information on our study observation and clarify the uncertainty on clinical benefits of platelet transfusion in dengue [9].

\section{Statement of Ethics}

Written informed consent was obtained from participants as required for a case series observation. Patient confidentiality was maintained as per national guidelines for research involving human participants.

\section{Disclosure Statement}

The authors have no conflicts of interest to declare. 


\section{References}

1 Lye DC, Archuleta S, Syed-Omar SF, Low JG, Oh HM, Wei Y, et al. Prophylactic platelet transfusion plus supportive care versus supportive care alone in adults with dengue and thrombocytopenia: a multicentre, open-label, randomised, superiority trial. Lancet. 2017 Apr;389(10079):1611-8.

2 Bhaskar E, Sowmya G, Moorthy S, Sundar V. Prevalence, patterns, and factors associated with bleeding tendencies in dengue. J Infect Dev Ctries. 2015 Jan;9(1):105-10.

3 Khan Assir MZ, Kamran U, Ahmad HI, Bashir S, Mansoor H, Anees SB, et al. Effectiveness of platelet transfusion in dengue $\mathrm{Fe}$ ver: a randomized controlled trial. Transfus Med Hemother. 2013 Oct;40(5):362-8.
4 Bolliger D, Seeberger MD, Tanaka KA. Principles and practice of thromboelastography in clinical coagulation management and transfusion practice. Transfus Med Rev. 2012 Jan; 26(1):1-13.

5 Schöchl H, Nienaber U, Hofer G, Voelckel W, Jambor C, Scharbert G, et al. Goal-directed coagulation management of major trauma patients using thromboelastometry (ROTEM)-guided administration of fibrinogen concentrate and prothrombin complex concentrate. Crit Care. 2010;14(2):R55.

6 Chavez JJ, Foley DE, Snider CC, Howell JC, Cohen E, Muenchen RA, et al. A novel thrombelastograph tissue factor/kaolin assay of activated clotting times for monitoring heparin anticoagulation during cardiopulmonary bypass. Anesth Analg. 2004 Nov;99(5):1290-4.
7 World Health Organization. Dengue: guidelines for diagnosis, treatment, prevention and control. Geneva: WHO; 2009 [accessed on 2018 July 3]. Available from: http://www. who.int/tdr/publications/documents/dengue-diagnosis.pdf.

8 Lee TH, Wong JG, Leo YS, Thein TL, Ng EL, Lee LK, et al. Potential Harm of Prophylactic Platelet Transfusion in Adult Dengue Patients. PLoS Negl Trop Dis. 2016 Mar; 10(3):e0004576

9 Israels SJ. Laboratory testing for platelet function disorders. Int J Lab Hematol. 2015 May; 37 Suppl 1:18-24. 